DISTRIBUTION STATEMENT A. Approved for public release; distribution is unlimited.

\title{
Improved EM Tactical Applications through UAS-Enhanced High-Resolution Mesoscale Data Assimilation and Modeling
}

\author{
Teddy R. Holt \\ Naval Research Laboratory \\ Monterey, CA 93943-5502 \\ phone: (831) 656-4740 fax: (831) 656-4769 e-mail: teddy.holt@nrlmry.navy.mil
}

Award Number: N0001413WX20927

\section{LONG-TERM GOALS}

The long-term goal of this project is to develop new and emerging analysis and forecast capabilities via improvements to environmental inputs to electro-magnetic (EM) tactical applications through Unmanned Aerial Systems (UAS) enhanced high-resolution mesoscale data assimilation and modeling. These capabilities will be achieved through the formulation, testing, evaluation, and demonstration of an environmental data assimilation and prediction system designed to quantify the UAS observation parameters, characteristics (e.g., vertical resolution, rate, etc.), measurement strategies and assimilation approaches that provide the greatest benefit for high-resolution (grid increment of 5-km or less) atmospheric tactical-scale environmental forecasts. The goal is to focus on EM tactical applications and metrics in evaluating the performance of the system. This development will leverage current and emerging capabilities for: i) environmental data assimilation, ii) adjoint-based sensitivity and observation impact, iii) air-ocean coupled modeling, and iv) EM tactical decision aid systems. Observational results from field programs such as Trident Warrior 2013 (TW13) will also be leveraged to build, test, and transition the prototype end-to-end (observation to tactical decision aid (TDA)) demonstration system. All meteorological inputs to the demonstration system will come from UAS platforms and sensors consistent with the present state-of-the-art; however, no specific operational UAS system will be targeted, transitioned or modified as part of this effort.

\section{OBJECTIVE}

The objective is to develop, validate, and transition new METOC capabilities within a high-resolution modeling system using advanced data assimilation of UAS data in order to achieve superior short and medium term forecasts of high-impact weather and EM-related parameters. We will use three crucial model components: i) the coupled atmospheric and oceanic components of the Coupled Ocean/Atmosphere Mesoscale Prediction System (COAMPS ${ }^{\circledR 1}$ ), ii) the 3D-variational (3DVAR) analysis schemes NAVDAS (NRL Atmospheric Variational Data Assimilation System) for the atmosphere and NCODA (Navy Coupled Ocean Data Assimilation) for the ocean and wave models, and iii) the COAMPS Adjoint observation impact system. COAMPS-OS ${ }^{\circledR 1}$ will be used as the software interface for configuring COAMPS and the other system components to facilitate high-resolution coupled mesoscale forecasts. The COAMPS system includes an ocean circulation model, the Navy

\footnotetext{
${ }^{1}$ COAMPS $^{\circledR}$ and COAMPS-OS ${ }^{\circledR}$ are registered trademarks of the Naval Research Laboratory.
} 


\section{Report Documentation Page}

Form Approved

OMB No. 0704-0188

Public reporting burden for the collection of information is estimated to average 1 hour per response, including the time for reviewing instructions, searching existing data sources, gathering and maintaining the data needed, and completing and reviewing the collection of information. Send comments regarding this burden estimate or any other aspect of this collection of information,

including suggestions for reducing this burden, to Washington Headquarters Services, Directorate for Information Operations and Reports, 1215 Jefferson Davis Highway, Suite 1204, Arlington

VA 22202-4302. Respondents should be aware that notwithstanding any other provision of law, no person shall be subject to a penalty for failing to comply with a collection of information if it

does not display a currently valid OMB control number.

1. REPORT DATE

30 SEP 2013

4. TITLE AND SUBTITLE

Improved EM Tactical Applications through UAS-Enhanced High-Resolution Mesoscale Data Assimilation and Modeling

6. AUTHOR(S)

7. PERFORMING ORGANIZATION NAME(S) AND ADDRESS(ES)

Naval Research Laboratory, 7 Grace Hopper

Avenue,Monterey,CA,93943-5502

9. SPONSORING/MONITORING AGENCY NAME(S) AND ADDRESS(ES)

\section{DATES COVERED}

00-00-2013 to 00-00-2013

5a. CONTRACT NUMBER

5b. GRANT NUMBER

5c. PROGRAM ELEMENT NUMBER

5d. PROJECT NUMBER

5e. TASK NUMBER

5f. WORK UNIT NUMBER

8. PERFORMING ORGANIZATION REPORT NUMBER

10. SPONSOR/MONITOR'S ACRONYM(S)

11. SPONSOR/MONITOR'S REPORT NUMBER(S)

12. DISTRIBUTION/AVAILABILITY STATEMENT

Approved for public release; distribution unlimited

13. SUPPLEMENTARY NOTES

14. ABSTRACT

15. SUBJECT TERMS

16. SECURITY CLASSIFICATION OF: a. REPORT

unclassified b. ABSTRACT unclassified c. THIS PAGE

unclassified
17. LIMITATION OF ABSTRACT

Same as

Report (SAR)
18. NUMBER 19a. NAME OF

OF PAGES

7 
Coastal Ocean Model (NCOM), which fully interacts with the nonhydrostatic atmospheric module. In addition, two different wave models are currently being implemented and tested: Simulating Waves Nearshore (SWAN) and Wavewatch III (WWIII) which will also be available for testing. NAVDAS is the Navy's state-of-the-art analyses system. The adjoint of the COAMPS forecast model and NAVDAS data assimilation systems have already been developed and linked together to provide a quantitative measure of the impact of individual observation types (e.g., radiosonde, aircraft, UAS, etc.) on the COAMPS forecast error. This observation impact capability has only recently become available for COAMPS and is one of the first such observation impact capabilities for a mesoscale modeling system developed anywhere.

Several key scientific objectives are identified in relation to the impact of the environment on EM propagation characteristics, particularly in the coastal marine atmospheric boundary layer (MABL): i) the time-varying 3-D structure of the MABL and its interaction and impact on EM propagation characteristics and ducting of EM energy, ii) the response of the MABL to the structure of the underlying SST, including SST gradients, and the impact on evaporative duct height calculations, on MABL refractive layers, and on larger-scale surface and MABL ducting phenomena, and iii) the most effective UAS observing strategies from an atmospheric model data assimilation perspective in order to accurately predict mesoscale weather and EM propagation conditions.

\section{APPROACH}

Our approach is to leverage prior investments in NRL-developed NAVDAS (including aircraft data assimilation), COAMPS, adjoint, and EM TDA technologies to produce a UAS-enabled, integrated, and automated on-scene system to support ASW, ASuW, and ISR mission planning and execution. Significant collaborations with other investigators developing and integrating UAS-based data and tools will be also leveraged. Collaborations with Scripps Institute of Oceanography (SIO), NSWCDahlgren, and other PMW-120 EM investigators will be pursued and help guide our sampling strategies, assure the UAS observations taken are in accord with NRL goals, and enhance synergistic development of UAS-based TDAs. Six specific technologies that will be developed, tested, and integrated in this project are i) tactical-scale data assimilation, ii) adjoint-based observation impact, iii) air-ocean coupled modeling, iv) EM tactical decision aid systems, v) software infrastructure, and vi) validation and verification of the end-to-end system.

\section{WORK COMPLETED}

Work that has been completed includes: i) development of UAS Scan Eagle (SE) data processing software (v1.0), ii) initial testing/implementation of SE data processing, iii) evaluation of pre-TW13 historical COAMPS forecasts, iv) successful support of TW13 field program (including 3DVAR assimilation of SE data in real time, development and evaluation of data assimilation innovation products highlighting differences between control and experiment runs using SE data, initial evaluation of high-resolution COAMPS real-time TW13 forecasts and adjoint forecasts, and initial evaluation of SE data impact on Advanced Refractive Effects Prediction System (AREPS) output.

i) Scan Eagle data processing software development: Software has been developed that includes a new SE observation decoder, modification of original UAS time averaging routines to average level 
and profile observations differently, implementation of FGAT (First Guess at Appropriate Time) software, and adjustment of data thinning parameters (used for all data).

ii) Scan Eagle data processing testing: SE UAS data processing software has been tested for an initial sample data set, and extended for TW13 data. Vertical profiles of wind speed and direction, air temperature, and water vapor have been evaluated for the lowest $\sim 1500-\mathrm{m}$ of the boundary layer. The sensitivity of vertical M-profiles to both slow- and fast-response data has been compared. Results show that fast-response data captures small-scale structures producing critically important local trapping regions often not resolved from slow-response data. AREPS has been evaluated using SE data as input for output parameters of $100 \mathrm{Mhz}$ VHF Radio frequency and X-band radar.

iii) COAMPS high-resolution simulations: Historical COAMPS simulations for July 2012 in the TW13 field program area (VACAPES) have been conducted and analyzed to characterize the climatological environment of the area. Results have been used to provide guidance for model setup in preparation for the field phase of TW13.

iv) TW13 field program support: Extensive modeling and operational support was provided for the July 2013 field program (see Results below).

\section{RESULTS}

\section{a. Scan Eagle data processing software development}

A significant amount of software development was made to the COAMPS data assimilation suite to incorporate UAS data. First, UAS observations pass through aircraft QC procedures originally developed for commercial aircraft observations. The UAS observations are elevation limited: observations far above the maximum height range of the UAS aircraft are automatically rejected; observations collected at 15-m elevation or lower are automatically rejected because the sensors are not shut off during landing/waiting for take-off. Second, because meteorological sensors and GPS sensors used different clocks, synchronization errors can produce unusable real-time winds. Thus, winds are not used (though wind errors can be corrected in post-processing). For the TW13 field program, the meteorological sensor clock was incorrectly set for part of the experiment (off by hours), causing all meteorological variables to be rejected because they were too "old". This time period was $\sim 00$ UTC 15 July to 00 UTC 16 July. Finally, NAVDAS performs additional data thinning (to remain computationally efficient), thinning data in areas where there are too many observations. So not all QC accepted SE data are used. Figure 1 describes the new data assimilation technique called First Guess at Appropriate Time (FGAT) that has been developed by NRL-MRY and tested and used with SE data for the TW13 field experiment. FGAT is a technique that allows data to be used at the most appropriate time within the data assimilation window for NAVDAS. The current FGAT implementation uses piecewise linear interpolation between the 3-, 6-, and 9-h forecast background fields. Results for the TW13 field experiment indicate that using FGAT provides improved analyses.

\section{b. Scan Eagle data processing testing}

Figure 2 shows the impact of using SE data in COAMPS analyses for the TW13 field program. Two parallel COAMPS simulations were conducted in real-time, one assimilating all routinely available 
data plus SE data (called tw13se) and one assimilating only routine data (tw13). Shown is the 1000$\mathrm{hPa}$ air temperature difference between two COAMPS nest-4 (1.33-km) analyses valid 12 UTC 16 July 2013 for TW13 (tw13se minus tw13). The triangles are locations of SE observations, with the innovations color coded the same as the temperature differences. Results indicate that NAVDAS makes the analysis using SE data (tw13se) warmer in the vicinity of the SE observations at this level $(1000-\mathrm{hPa})$ for this analysis time. This is in agreement with the positive analysis temperature difference (tw13se - tw13). Differences further away from SE observation locations are the result of accumulating differences between analyses (and model background fields based on the 6-h forecasts) over the course of the experiment.

\section{c. COAMPS adjoint sensitivity}

An adjoint allows one to compute the sensitivity of a particular forecast output (e.g. the modified refractivity in the lowest $200 \mathrm{~m}$ ) to changes in the initial state in a mathematically rigorous and computationally feasible manner. The COAMPS adjoint identifies regions of greatest sensitivity in the initial state and highlights the areas where additional observations (targeting) could improve the forecast the most. In preparation for the TW13 field campaign, the first-ever COAMPS adjoint simulations using the vertical gradient of modified refractivity $(\mathrm{M})$ were conducted and evaluated for the TW13 experiment area. We have computed the adjoint sensitivity response function of the 6-h forecast "M-profile" modified refractivity to initial time vertically integrated kinetic energy as well as initial time sea surface temperature for various test periods. Results from this example shows how forecasted refractivity in an area of interest projects back to where SST or wind speed observations should be taken, as well as the UAS proposed flight profile within range from R/V Knorr. Using this knowledge we employed the COAMPS nested adjoint in real-time for the TW13 field program. Figure 3a shows the COAMPS nest-4 (1.33-km) adjoint sensitivity response function of the 3-h forecast vertical gradient of modified refractivity (red box IVO R/V Knorr) to initial time vertically integrated total energy for 00 UTC 16 July 2013. Regions of large sensitivity occur in the vicinity of the Knorr, and initial condition sensitivity is a maximum in the MABL. The variable of greatest sensitivity is moisture (most important variable to observe). If the optimal perturbation was included, there could be a large impact on EM propagation, as illustrated in the modified refractivity profiles valid 09 UTC 16 July (Fig. 3b). Note that the profile with the adjoint optimal interpolation (red line) indicates a ducting regime that does not occur for the control (without perturbation).

\section{d. SE data impact on AREPS output}

Currently there exist several EM TDAs. AREPS is the Navy's sensor and communications prediction software system, providing radar probability of detection and communications assessments for onboard sensors operating in coastal, ocean and littoral environments. Refractive effects from these mesoscale-rich environments are introduced through ingest of NWP model forecast fields yielding vertical modified index of refraction (M)-profiles and propagation condition summaries along with the height-range propagation loss for multiple bearings. Figure 4 shows an example from the TW13 field experiment illustrating the impact of using SE data on EM propagation. Figure 4a shows a map of the location of transmitter (WYSJ-TV Yorktown) and R/V Knorr used for calculations of EM propagation. Point propagation summaries for the Knorr balloon launch valid 0933 UTC 16 July and the two 18 UTC 15 July 15-h COAMPS nest-4 (1.33-km) forecasts (tw13 and tw13se) are shown in Fig. 4b. Results indicate similar duct thicknesses between the observed balloon launch and the COAMPS 
forecasts. Note that the magenta region for the balloon and the red regions for the COAMPS simulations show good agreement (thickness $\sim 200-\mathrm{m}$ ). The range dependent propagation summaries are shown in Fig. 4c along the WYSJ-Knorr path, continuing offshore for $\sim 250-\mathrm{km}$. The forecast for tw13 indicates a coastal trapping layer that increases with height (indicated by red line in top panel) that is more prominent than for tw13se (bottom panel). A second trapping layer exists for both forecasts at $\sim 110-\mathrm{km}$ downrange to $\sim 250-\mathrm{km}$. The associated propagation loss coverage diagrams for the three vertical profiles along the WYSJ-Knorr path is shown in Fig. 5. There is similar agreement between the balloon and both COAMPS forecasts from WYSJ to Knorr downrange to $\sim 60-\mathrm{km}$, but more loss at the Knorr in the lowest $\sim 100$-m for tw13se and more loss beyond Knorr for tw13 ( $>\sim 100$ $\mathrm{km}$ ) (as indicated by the dashed white ovals).

\section{RELATED PROJECTS}

COAMPS will be used in related 6.1 projects within PE $0601153 \mathrm{~N}$ that include studies of air-ocean coupling and boundary layer studies, and in related 6.2 projects within PE $0602435 \mathrm{~N}$ that focus on the development of the atmospheric components (QC, analysis, initialization, and forecast model) of COAMPS.

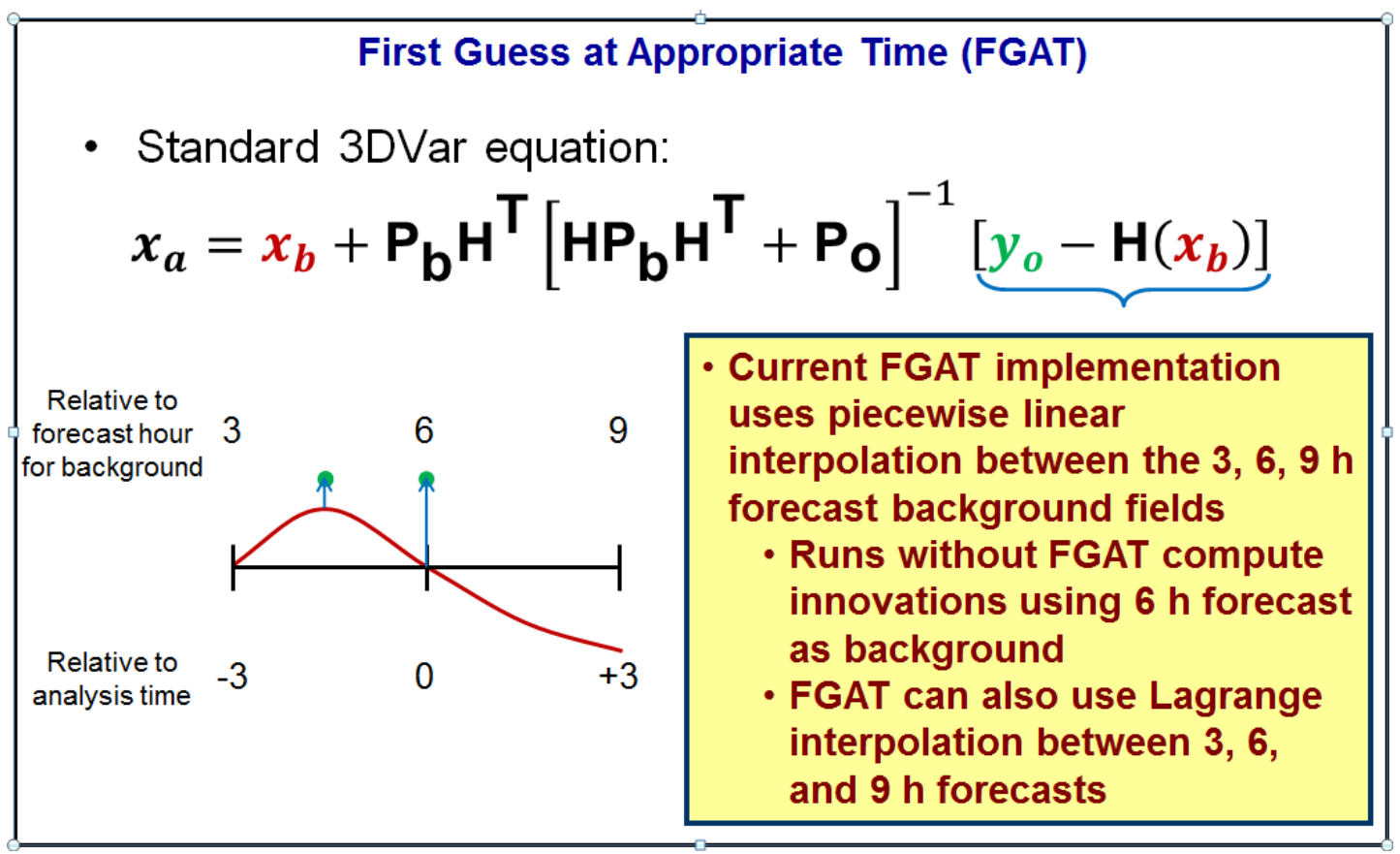

Figure 1. Description of the new First Guess at Appropriate Time (FGAT) technique. 


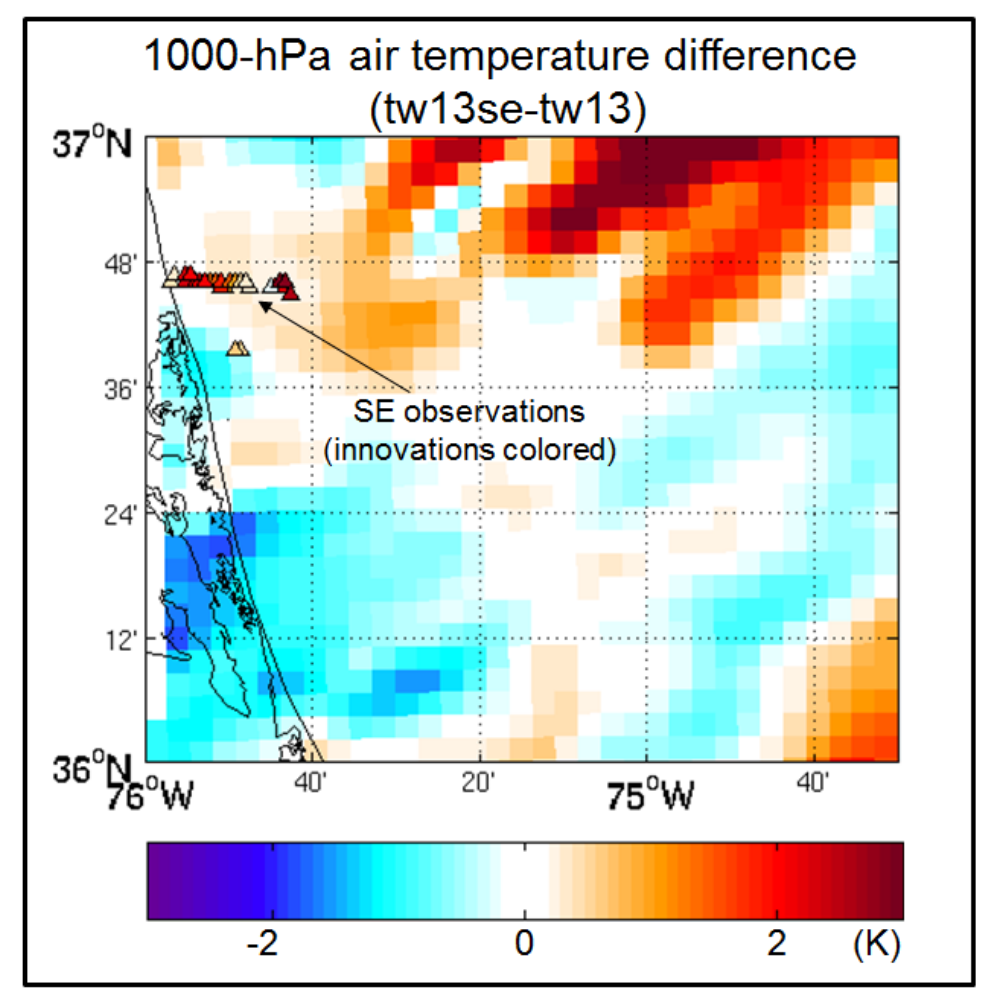

Figure 2. 1000-hPa air temperature difference between two COAMPS nest-4 (1.33-km) analyses valid 12 UTC 16 July 2013 for TW13 (COAMPS with SE data minus COAMPS without SE data). The triangles are locations of $S E$ observations, with the innovations color coded the same as the temperature differences.

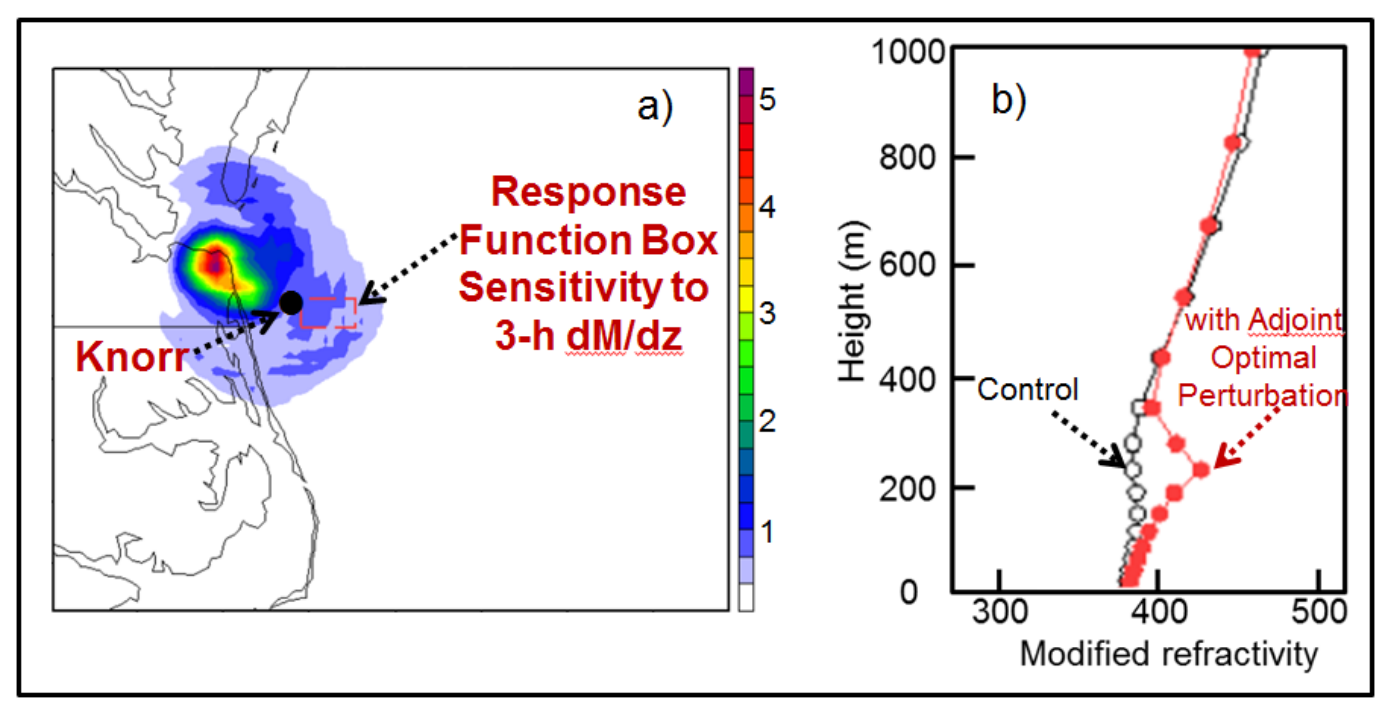

Figure 3. a) COAMPS nest-4 (1.33-km) adjoint sensitivity response function of the 3-h forecast vertical gradient of modified refractivity (red box IVO R/V Knorr) to initial time vertically integrated total energy for 00 UTC 16 July 2013, and b) modified refractivity profiles valid 09 UTC 16 July for the control (black line, without perturbation) and with the adjoint optimal interpolation (red line). 


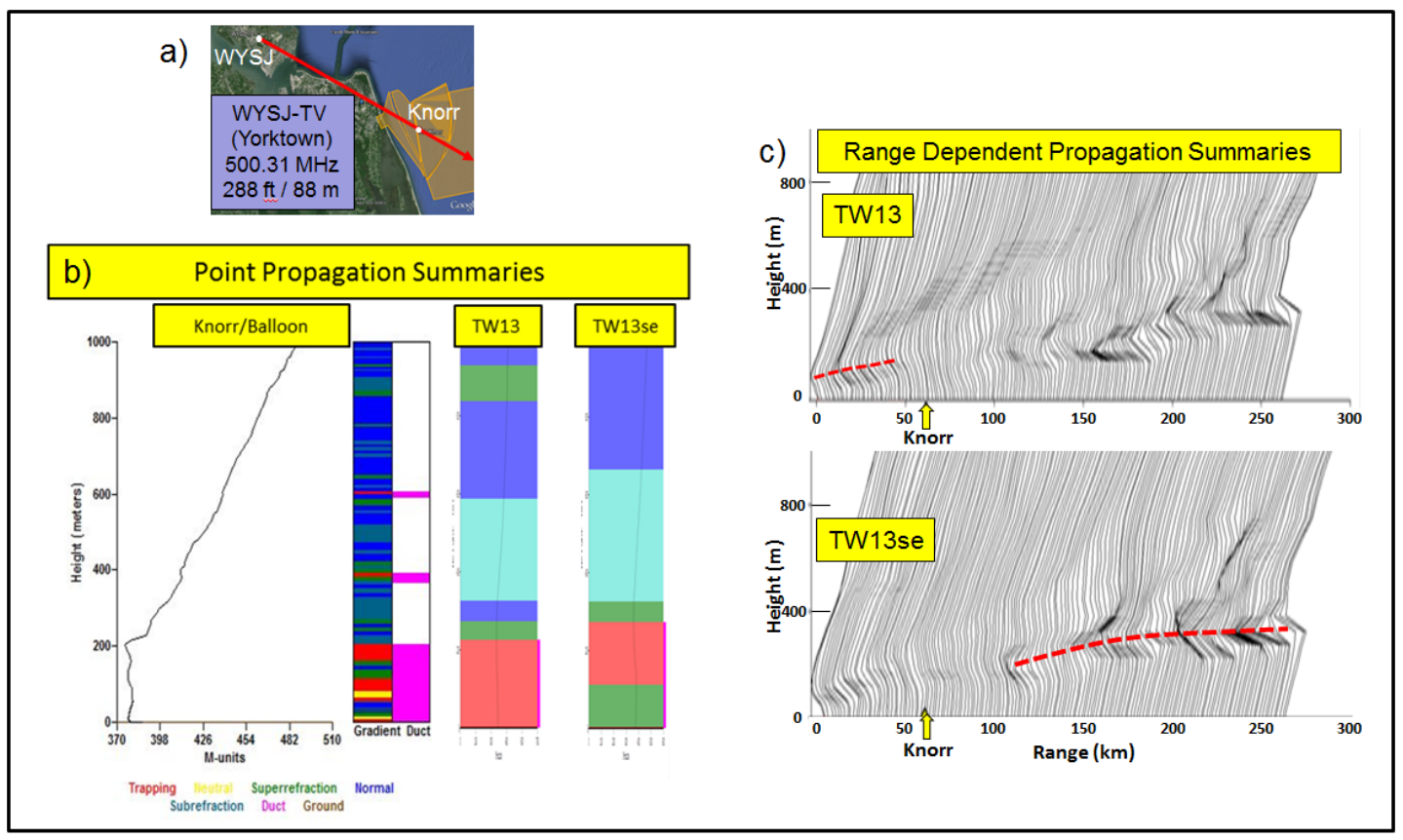

Figure 4. a) Map of location of transmitter (WYSJ-TV Yorktown) and R/V Knorr used for calculations of EM propagation, b) point propagation summaries for the Knorr balloon launch valid 0933 UTC 16 July (left) and the two 18 UTC 15 July 15-h COAMPS nest-4 (1.33-km) forecasts (tw13 and tw13se), and c) range dependent propagation summaries along the WYSJ-Knorr path, continuing offshore for $\sim 250-\mathrm{km}$.

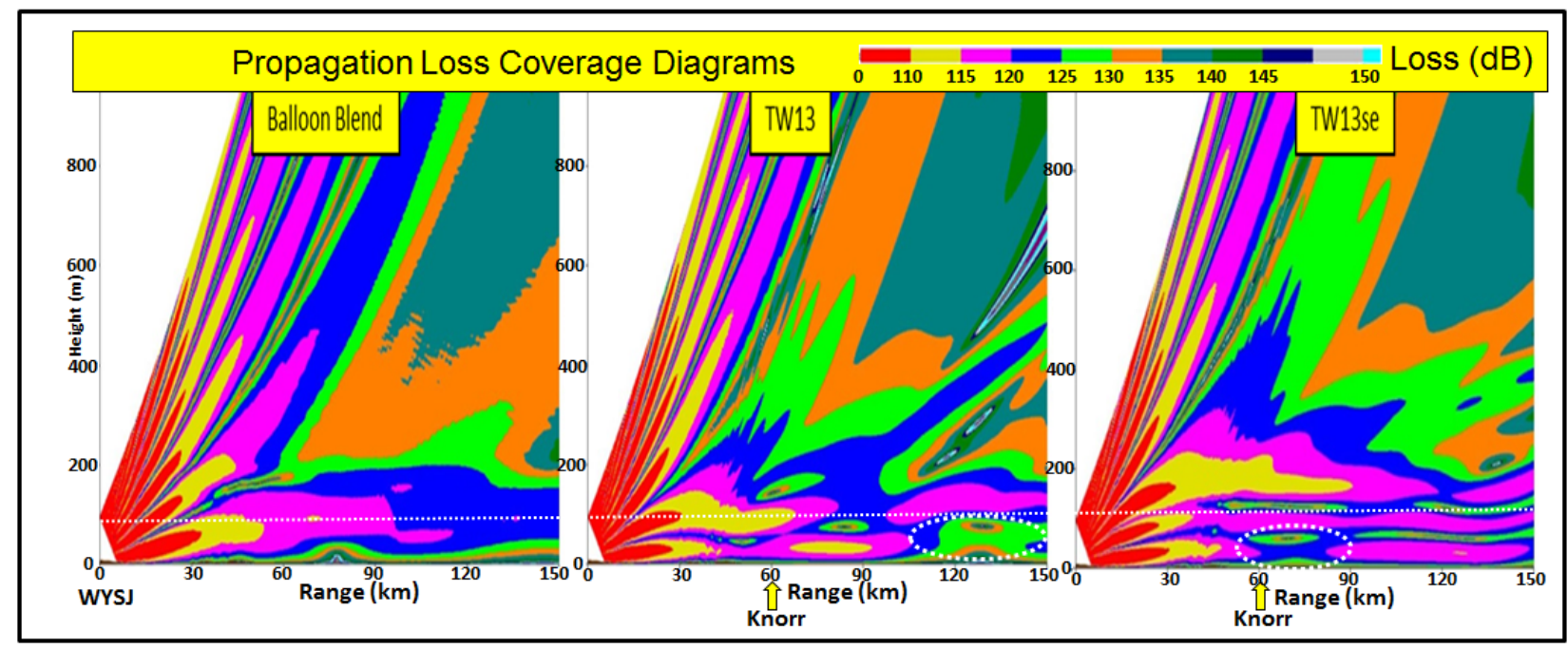

Figure 5. Propagation loss diagrams for the Knorr balloon launch (left) as described in Fig. 4, and the COAMPS forecasts (tw13 and tw13se). 